\title{
Safety of stress testing in patients with elevated cardiac biomarkers: Are all modalities created equal?
}

\author{
Rami Doukky, MD, MSc, FACC, FASNC, ${ }^{\mathrm{a}, \mathrm{b}}$ and Yasmeen Golzar, MD, FACC \\ a Division of Cardiology, John H. Stroger, Jr. Hospital of Cook County, Chicago, IL \\ ${ }^{b}$ Division of Cardiology, Rush University Medical Center, Chicago, IL
}

Received Feb 2, 2016; accepted Feb 2, 2016

doi: 10.1007/s12350-016-0440-4

\section{See related article, pp. 724-734}

The safety of stress radionuclide myocardial perfusion imaging (MPI) was established more than two decades ago. ${ }^{1-3}$ This supposition has been a solid foundation in our practice such that we often resort to exercise and pharmacologic stress studies as the preferred risk stratification tools and as means to mitigate risk associated with invasive procedures. Before invasive coronary angiography was widely implemented in the evaluation of patients recovering from uncomplicated myocardial infarction (MI), pre-discharge lowlevel exercise stress test, using the modified Bruce protocol, was established as a safe and effective risk stratification tool. Seminal work by leaders in the field of nuclear cardiology capitalized on the added prognostic value of MPI to enhance the risk assessment of patients recovering from uncomplicated MI. Heller et al and Brown et al demonstrated that dipyridamole MPI performed 2-4 days following uncomplicated MI is not only safe but also superior to submaximal exercise stress MPI in identifying patients at risk. ${ }^{4,5}$ On the other hand, Mahmarian et al demonstrated the added prognostic value of adenosine stress MPI to invasive coronary angiography in patients with uncomplicated MI. ${ }^{6}$ Such studies paved the way for the INSPIRE trial which prospectively demonstrated that adenosine stress radionuclide MPI, performed within 10 days of

Reprint requests: Rami Doukky, MD, MSc, FACC, FASNC, Division of Cardiology, John H. Stroger, Jr. Hospital of Cook County, 1901 W. Harrison St., Suite \# 3620, Chicago, IL 60612; rami_doukky@rush.edu J Nucl Cardiol 2017;24:735-7.

$1071-3581 / \$ 34.00$

Copyright (C) 2016 American Society of Nuclear Cardiology. uncomplicated MI, not only defines risk but can also guide patient management. ${ }^{7}$

Supported by robust outcome data, the safety of post-MI vasodilator stress MPI went unchallenged for years. When regadenoson was introduced in the U.S., many practitioners extrapolated from dipyridamole and adenosine literature and used the new agent in the risk stratification of post-MI patients. A growing body of literature establishing the prognostic value of regadenoson radionuclide MPI as an equivalent to adenosine stress soon followed. ${ }^{8-11}$ However, to date, there has not been a large study affirming the safety of regadenoson in post-MI patients or those with elevated cardiac troponin levels. The question can be asked: since they are similar in clinical utility, why cannot we extrapolate the safety profile of adenosine to regadenoson? There are important pharmacodynamic and pharmacokinetic differences between adenosine and regadenoson. The half-life of adenosine is extremely short ( $<10$ seconds); thus, its hemodynamic effects end promptly after the termination of infusion. On the other hand, regadenoson has more complex pharmacokinetics, as it displays its effect in three phases and half-lives: an initial phase is approximately 2 to 4 minutes; an intermediate phase follows, with a half-life of 30 minutes coinciding with loss of the pharmacodynamic effect; and the terminal phase consists of a decline in plasma concentration with a half-life of approximately 2 hours. In addition to a longer halflife, the pharmacodynamics of regadenoson is slightly different, manifesting with a greater increase in heart rate. ${ }^{12}$ This prolonged chronotropic response may lead to an extended period of ischemia among patients with severe coronary artery disease, which may be particularly detrimental among those presenting with acute coronary syndrome. ${ }^{13}$ In fact, several reports of MI during regadenoson stress have surfaced in the literature and the FDA Adverse Event Reporting System resulting in changes to the drug labels of both adenosine and 
regadenoson over concerns of increased risk of MI and death. ${ }^{14}$ With millions of radionuclide MPI studies being performed annually in the U.S., addressing the safety profile of various stress testing modalities, particularly in post-MI patients, is paramount. Moreover, when it comes to the safety of stress MPI in patients with uncomplicated MI, were various stress modalities created equal?

In this issue of the journal, Rai et $\mathrm{al}^{15}$ revisited and compared the safety of vasodilator stress (regadenoson, dipyridamole, and adenosine) in patients with elevated cardiac troponin. In a retrospective design, the authors reviewed 703 consecutive stress MPI studies of patients with elevated cardiac troponin $\leq 7$ days prior to testing at two academic centers. In the process, the authors reported on patients who underwent dobutamine and exercise stress MPI in the same setting. The study population, patients with elevated cardiac troponin $\leq 7$ days prior to testing, represents a growing challenge in modern practice; with increasing sensitivity of cardiac troponin assays, more patients are found to have elevated cardiac troponins who do not exactly fit the classic clinical scenario of acute coronary syndrome. The study population is likely composed of a heterogeneous mix of patients: recent myocardial infarction; clinical syndromes associated with increased myocardial oxygen demand, such as hypertensive crisis, tachyarrhythmias, and heart failure; and other patients with unexplained elevation of cardiac troponin. Notably, patients with confirmed recent MI who were selected to undergo stress MPI are likely to have been perceived to be "high-risk" for invasive coronary angiography, likely due to chronic kidney disease or bleeding tendencies. Adverse events within 24 hours of stress MPI were obtained from the stress test reports and electronic medical records. The primary endpoint was a composite of death, non-fatal MI, new or worsening heart failure, stroke, ventricular arrhythmias, atrial fibrillation/flutter, or atrioventricular block requiring intervention. The majority of the patients $(90 \%)$ underwent vasodilator stress: $51 \%$ regadenoson, $28 \%$ dipyridamole, and $11 \%$ adenosine. Only $9(1 \%)$ and 61 (9\%) subjects underwent dobutamine and exercise stress, respectively. For vasodilator stress modalities, TIMI scores were significantly higher and the studies were performed slightly later (mean 3.1 days) after elevated troponin than for patients who underwent exercise protocols (mean 2.0 days).

The incidence of adverse events was low in all vasodilator stress groups with the composite endpoint ranging from $1.0 \%$ to $1.4 \%$. The highest incidence of the composite endpoint was in the dobutamine stress group at $11.1 \%$; the second highest was in the exercise stress group at $3.3 \%$. The event rates for dobutamine and exercise stress should be interpreted with caution since only 9 and 61 patients, respectively, underwent these stress modalities. The most frequent adverse event was non-fatal MI which occurred in $7(1.0 \%)$ patients [0.8\%, regadenoson; $0 \%$, dipyridamole; $1.4 \%$, adenosine; $11.1 \%$ dobutamine; $3.3 \%$ exercise stress $(P=.005)]$. The study is markedly underpowered to detect differences in safety profile between vasodilator stress and other stress modalities, and even more underpowered to detect safety differences among vasodilators stress agents. Furthermore, given the small sample size and number of events, any univariate or multivariate analysis trying to identify patients at risk for adverse events is fraught with problems due to multiple testing and model overfitting.

Nonetheless, the study findings are timely and important in a few respects. First, it demonstrates that risk of adverse events with vasodilator stress in patients with elevated cardiac troponin is low but not negligible (1.0-1.4\%). Second, there is no demonstrable difference in risk of adverse events between older vasodilator stress agents (dipyridamole and adenosine) and regadenoson. If any differences exist, they are too small to be observed in a study of a few hundred subjects and thus are unlikely to be clinically significant. Third, there may be a trend towards increased event rates in patients undergoing dobutamine and exercise stress. Although, the sample size and number of events are too small to draw any conclusions, these observations are consistent with our understanding of the pharmacologic and physiologic effects of dobutamine and exercise. Both of these modalities lead to an uncontrolled increase in heart rate and blood pressure, leading to an increase in myocardial oxygen demand; this is often prolonged in the case of dobutamine (half-life 2 minutes). ${ }^{16}$ In a previous era, the modified Bruce protocol was the standard pre-discharge risk stratification tool of post-MI patients, postponing symptom-limited standard Bruce protocol several weeks after discharge. ${ }^{4,5}$ The present work by Rai et al reminds us that maximal stress protocol using dobutamine stress or standard Bruce protocol may not be appropriate for high-risk patients recovering from uncomplicated MI.

It is important to note that some of the events observed in the present study were destined to happen whether the patient underwent stress testing or not due to "background" incidence of adverse events in this high-risk population. Since the study did not include a control group of patients with elevated cardiac troponin who did not undergo stress testing, it is impossible to attribute causality of adverse events to stress testing in some of these cases.

With a growing emphasis being placed on expeditious inpatient evaluations and shortened hospital stays, 
we sometimes forget that stress testing can occasionally lead to serious complications. ${ }^{17}$ It is important to ask: are these tests safe in post-MI patients and those with elevated cardiac troponin levels? Is the newer vasodilator stress agent regadenoson as safe as its predecessors? Despite some limitations, the study by Rai et al provides timely, though incomplete, answers to these questions. With high-sensitivity cardiac troponin assays on the horizon, more patients with elevated cardiac troponin who do not fit the typical paradigm of acute coronary syndrome will present to our stress laboratories. This study adds to the body of literature affirming the relative safety of all vasodilator stress agents and poses the challenge of building a large registry of patients undergoing pharmacologic stress to tease out any safety differences between these agents.

\section{Disclosures}

Rami Doukky receives research funding from Astellas Pharma and served on the Advisory Board for Astellas Pharma.

\section{References}

1. Lette J, Tatum JL, Fraser S, Miller DD, Waters DD, Heller G, et al. Safety of dipyridamole testing in 73,806 patients: The Multicenter Dipyridamole Safety Study. J Nucl Cardiol 1995;2:317.

2. Cerqueira MD, Verani MS, Schwaiger M, Heo J, Iskandrian AS. Safety profile of adenosine stress perfusion imaging: Results from the Adenoscan Multicenter Trial Registry. J Am Coll Cardiol 1994;23:384-9.

3. Elhendy A, Valkema R, van Domburg RT, Bax JJ, Nierop PR, Cornel JH, et al. Safety of dobutamine-atropine stress myocardial perfusion scintigraphy. J Nucl Med 1998;39:1662-6.

4. Brown KA, Heller GV, Landin RS, Shaw LJ, Beller GA, Pasquale MJ, et al. Early dipyridamole $(99 \mathrm{~m}) \mathrm{Tc}$-sestamibi single photon emission computed tomographic imaging 2 to 4 days after acute myocardial infarction predicts in-hospital and postdischarge cardiac events: Comparison with submaximal exercise imaging. Circulation 1999;100:2060-6.

5. Heller GV, Brown KA, Landin RJ, Haber SB. Safety of early intravenous dipyridamole technetium 99m sestamibi SPECT myocardial perfusion imaging after uncomplicated first myocardial infarction. Early Post MI IV Dipyridamole Study (EPIDS). Am Heart J 1997;134:105-11.
6. Mahmarian JJ, Mahmarian AC, Marks GF, Pratt CM, Verani MS. Role of adenosine thallium-201 tomography for defining longterm risk in patients after acute myocardial infarction. J Am Coll Cardiol 1995;25:1333-40.

7. Mahmarian JJ, Shaw LJ, Filipchuk NG, Dakik HA, Iskander SS, Ruddy TD, et al. A multinational study to establish the value of early adenosine technetium-99m sestamibi myocardial perfusion imaging in identifying a low-risk group for early hospital discharge after acute myocardial infarction. J Am Coll Cardiol 2006;48:2448-57.

8. Mahmarian JJ, Peterson LE, Xu J, Cerqueira MD, Iskandrian AE, Bateman TM, et al. Regadenoson provides perfusion results comparable to adenosine in heterogeneous patient populations: A quantitative analysis from the ADVANCE MPI trials. J Nucl Cardiol 2015;22:248-61.

9. Farzaneh-Far A, Shaw LK, Dunning A, Oldan JD, O'Connor CM, Borges-Neto S. Comparison of the prognostic value of regadenoson and adenosine myocardial perfusion imaging. J Nucl Cardiol 2015;22:600-7.

10. Doukky R, Fughhi I, Campagnoli T, Wassouf M, Ali A. The prognostic value of regadenoson SPECT myocardial perfusion imaging in patients with end-stage renal disease. J Nucl Cardiol 2015.

11. Hage FG, Ghimire G, Lester D, McKay J, Bleich S, El-Hajj S, et al. The prognostic value of regadenoson myocardial perfusion imaging. J Nucl Cardiol 2015;22:214-21.

12. Iskandrian AE, Bateman TM, Belardinelli L, Blackburn B, Cerqueira MD, Hendel RC, et al. Adenosine versus regadenoson comparative evaluation in myocardial perfusion imaging: Results of the ADVANCE phase 3 multicenter international trial. J Nucl Cardiol 2007; 14:645-58.

13. Doukky R, Olusanya A, Vashistha R, Saini A, Fughhi I, Mansour $\mathrm{K}$, et al. Diagnostic and prognostic significance of ischemic electrocardiographic changes with regadenoson-stress myocardial perfusion imaging. J Nucl Cardiol 2015;22:700-13.

14. Hage FG, Iskandrian AE. Serious complications associated with regadenoson administration for myocardial perfusion imaging: A commentary. J Nucl Cardiol 2014;21:877-9.

15. Rai M, Ahlberg AW, Marwell J, Chaudhary W, Savino JA, III, Alter EL et al. Safety of vasodilator stress myocardial perfusion imaging in patients with elevated cardiac biomarkers. J Nucl Cardiol 2016;23.

16. Doukky R. Pharmacologic Stress testing in myocardial perfusion imaging: Technical applications. In: Mann A, Heller GV, Hendel RC, editors. Nuclear Cardiology: Technical Applications. New York; 2007. p. 107-24.

17. Dilsizian V, Gewirtz H, Paivanas N, Kitsiou AN, Hage FG, Crone NE, et al. Serious and potentially life threatening complications of cardiac stress testing: Physiological mechanisms and management strategies. J Nucl Cardiol 2015;22:1198-213. 